\title{
Extinction persistence in the rat following brief training with constant or partial delay of reward
}

\author{
PATRICK E. CAMPBELL \\ Wright State University, Dayton, Ohio 45431 \\ MARK CLINE \\ Southern Methodist University, Dallas, Texas 75275
}

\begin{abstract}
Forty-eight rats were trained in a runway under three acquisition schedules: constant immediate reward, partial delay of reward, and constantly delayed reward. Delay occurred in the goalbox for a duration of $20 \mathrm{sec}$. Acquisition trials were given at one trial a day for 10 days. The constant delay group ran somewhat slower in acquisition than the other two groups. During extinction there were two treatment levels; constant confinement and no confinement. Confinement occurred in the goalbox for $20 \mathrm{sec}$, and one extinction trial was given each day for 24 days. The animals which were confined during extinction ran significantly slower than the nonconfined animals. The extinction effects of the acquisition schedules included a modest partial delay extinction effect but no evidence that constant delay caused an increase in persistence.
\end{abstract}

It is quite widely accepted that intermittent delays during acquisition tend to increase the persistence of an instrumental response during extinction. There is much less agreement, however, concerning the extinction effect of constant delay of reinforcement. For example, Renner (1964), in an early review, concluded that "more experimental work is needed to first demonstrate conclusively if delay of reinforcement during acquisition is sufficient to produce resistance to extinction or if it is the partial or variable aspect that is necessary"(p. 350). Shortly after the publication of the Renner review, McCain and Bowen (1967) reported an experiment that seems to support the idea that constant delay increases persistence relative to a no-delay control. Their experiment involved an extensive (12-day) pretraining period followed by a single day of acquisition training. Twenty animals were given four immediately rewarded acquisition trials and 20 were given four trials with a constant 20-sec delay. On the following day, all animals were given one additional acquisition trial and 13 extinction trials. The animals were confined to the goalbox for $20 \mathrm{sec}$ on each extinction trial. During acquisition, both groups showed a general increase in running speed but no differences between the groups were apparent. During extinction, however, the delayed animals were superior to the nondelayed rats, and their relative superiority increased over trials. These data clearly suggest that constant delay can result in greater extinction persistence than immediate reward.

Request for reprints should be sent to Patrick E. Campbell, Department of Psychology, Wright State University, Dayton, Ohio 454.31 .
One purpose of the present experiment was to provide a systematic replication of the McCain and Bowen experiment. The large majority of constant delay experiments have not observed any increased persistence associated with constant delay (cf. Campbell \& Knouse, 1972). Perhaps McCain and Bowen have reported a reliable phenomenon, but it is simply limited to the brief training situation. All of the contrary studies in the earlier reviews (Campbell \& Knouse, 1972; Renner, 1964) carried acquisition considerably beyond the five trials reported by McCain and Bowen. An additional possibility is that the McCain and Bowen finding is reliable but restricted to the situation where extinction confinement is held constant at a fairly high level. Recall that all of their animals were confined for $20 \mathrm{sec}$ during extinction. This procedure resulted in overall increase in confinement for the immediately rewarded rats but no change in the confinement for the delayed rats. Several experiments (cf. Campbell \& Knouse, 1972) have shown that extinction confinement is an important determinant of persistence in constant delay studies. The present experiment included the extinction confinement variable as well as the acquisition delay variable.

As we mentioned earlier, the issue as to whether the partial delay extinction effect is reliable is seldom raised. Nevertheless, the interpretation of nearly all partial delay experiments is open to argument since the extinction confinement variable is quite generally ignored in such experiments. As a result, partially delayed subjects experience an overall decrease in confinement with the switch from acquisition to nonconfined extinction, while the nondelayed subjects experience no change in confinement. Wike, Mellgren, 


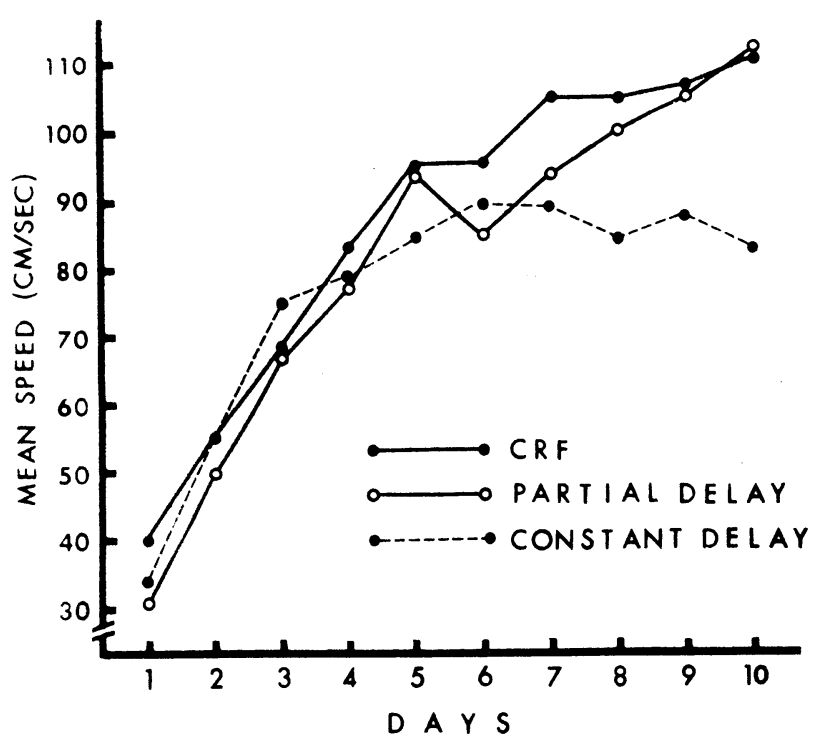

Figure 1. Total alley speeds for each schedule during acquisition.

and Wike (1968) have already commented on this generally ignored source of confounding and have provided data suggesting that decreases in confinement from acquisition to extinction increases persistence while increases in confinement from acquisition to extinction tend to decrease persistence. The present experiment included the extinction variable in a factorial arrangement with acquisition delay.

\section{METHOD}

The subjects were 48 experimentally naive male albino rats of the Sprague-Dawley strain. The animals were approximately 90 days old at the beginning of the experiment.

The apparatus was a $182 \times 15 \times 9 \mathrm{~cm}$ runway painted flat black throughout and covered with hinged clear plastic tops. Start, run, and goal times were measured photoelectrically over 25-, 100-, and 25-cm sections of the alley. The goal area was separated from the remainder of the alley by a manually operated guillotine door. A masking noise (white) of approximately $85 \mathrm{~dB}$ (SPL) was presented through three speakers mounted at equal spaces under the alley. Three $25-\mathrm{W}$ red lamps were placed approximately $2 \mathrm{ft}$ over the alley and provided the only source of illumination during training.

The experiment was conducted in two replications of 24 subjects each. One subject from each replication died during training. During 8 days of pretraining, the rats were reduced to approximately $85 \%$ of their normal body weights by being fed a daily ration of $5 \mathrm{~g}$ during pretraining and $15 \mathrm{~g}$ during the remainder of the experiment. Water was continually available in the home cages. Since only a brief training period was planned, the animals were given a rather elaborate taming regimen. During the first 4 days, the animals were handled extensively and trained to eat $97-\mathrm{mg}$ Noyes pellets. The last 4 days included additional handling and several unrewarded exploration trials in the runway.

Acqusition training consisted of 10 rewarded trials given at a rate of one each day. Each trial was completed with a five-pellet reward. One-third of the animals received the reward immediately after entering the goalbox on each trial (no delay). Another third of the rats were given immediate reward on all even-numbered days and a 20 -sec delay on all odd days (partial delay). The remaining animals were delayed for $20 \mathrm{sec}$ before being reinforced on every trial (constant delay). All delays occurred in the goalbox and were accomplished by screening off the goal cup and confining the subject to the goal area during the delay interval.

Extinction trials were also given at only one trial a day but were continued for 24 days. One-half of the animals within each acquisition condition were confined in the goal area for $20 \mathrm{sec}$ on each extinction trial. The remaining animals were not confined during extinction. A confined extinction trial was procedurally identical to a delayed trial except, of course, no food was delivered at the end of the interval.

The design of the present experiment was a 3 (acquisition schedule) by 2 (extinction schedule) by 2 (replications) factorial design. For the purpose of statistical analyses, a fourth variable (blocks of trials) was added to the design as a repeated measures factor.

\section{RESULTS}

The response times were converted to a speed measure (centimeters/second) following the typical procedure in runway experiments. Since the results from the different alley sections were quite similar, only the total alley measure will be presented here.

Figure 1 presents the acquisition data for the three schedules on each day of acquisition training. Inspection of the figure suggests that early in training the animals did not differ greatly, but, as training continued, the animals trained with the constant delay schedule showed some suppression of running speed. An analysis of variance confirmed these suggestions. Reward schedule was not significant as a main effect $(F=1.38, \mathrm{df}=2 / 40$, $\mathrm{p}>.263$ ), but did show a reliable interaction with trials $(F=1.87, d f=9 / 360, p<.017)$.

Figure 2 presents the extinction speeds for each acquisition schedule in blocks of four trials. These acquisition schedule main effects are collapsed across the extinction confinement variable. Figure 3 shows the extinction speeds as a function of the confinement

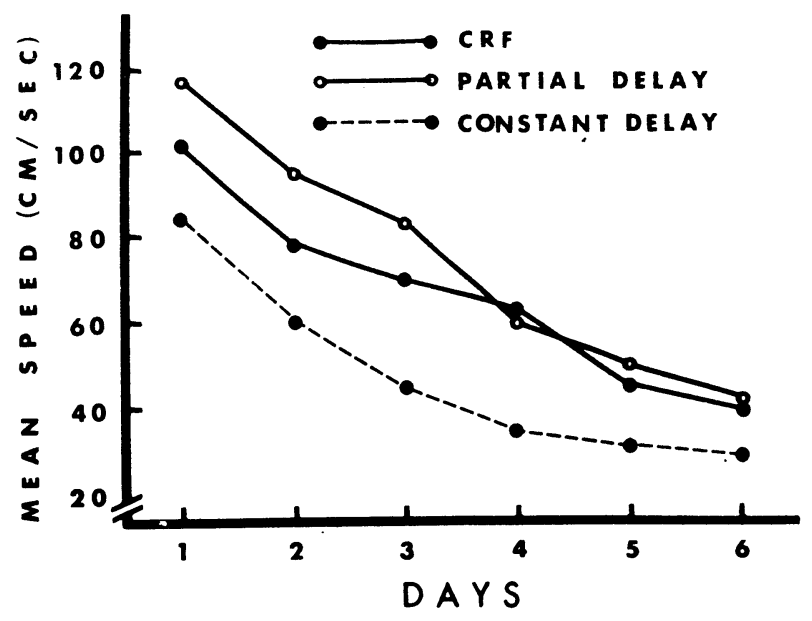

Figure 2. Extinction total alley speeds for each acquisition schedule. 
variable. As Figure 3 clearly suggests, the animals confined during extinction ran more slowly than the animals not confined. An analysis of variance confirmed that confinement was significant as a main effect $(\mathrm{F}=13.56, \mathrm{df}=1 / 34, \mathrm{p}<.0008)$ and as an interaction with trials $(\mathrm{F}=4.13, \mathrm{df}=5 / 170, \mathrm{p}<.0014)$. Figure 2 shows that the constant delay animals ran somewhat more slowly than the other rats, but all three groups show approximately equal rates of extinction (i.e., equal slopes for the three curves). Again, an analysis of variance confirmed these suggestions. Schedule proved to be significant as a main effect $(F=7.03$, $\mathrm{df}=2 / 34$, $\mathrm{p}<.003)$ but not as an interaction with trials $(F=1.31$, $\mathrm{df}=10 / 170, \mathrm{p}>.229)$. The absence of $\mathrm{a}$ schedule-by-trials interaction, of course, suggests that the three groups extinguished with equal rates although the speeds were different.

Figure 2 also shows a slight suggestion of a partial delay extinction effect. That is, the partial delay rats ran somewhat faster than the nondelayed rats during the first 12 days of extinction (Blocks 1-3). An LSD statistic showed this effect to be significant $(p<.05)$ when the first three blocks are averaged together. The LSD test was selected since a partial delay extinction effect could be considered an a priori hypothesis.

Finally, the acquisition schedule by extinction confinement interaction (data not shown) was negligible $(\mathrm{F}=.106, \mathrm{df}=2 / 34, \mathrm{p}>.899)$ as was the three-factor interactive involving these two variables with trials $(\mathrm{F}=.99, \mathrm{df}=10 / 170, \mathrm{p}>.455)$. The absence of these interactions, of course, suggests that either of the two variables may be meaningfully considered without consideration of the other.

\section{DISCUSSION}

The acquisition results from the present experiment followed the typical pattern of similar experiments. Our findings that constant delay but not partial delay caused slower acquisition speeds are consistent with findings of several studies reviewed by Renner (1964) and by Campbell and Knouse (1972).

The results of the extinction phase were also consistent with most available evidence. For example, the short-trial partial delay effect reported by Howlett and Sheldon (1968) was successfully replicated. The size of this effect, however, was quite meager when compared with the typical extended-trial partial delay effect, or the brief-trial partial reinforcement effect reviewed by Robbins (1971).

The suggestion of Wike et al. (1968) that the confinement variable would show an important interaction with the partial delay aspect of acquisition training was not supported by the results of the experiment. We could find no evidence for such an interaction. As a result, our earlier concern that the partial delay extinction effect might be an artifact of shifting to extinction without confinement is no longer warranted. Further experiments of a partial delay nature can elect to hold the confinement variable constant and not expend the extra effort required to factor confinement trials, unless, of course, extinction confinement is a variable of interest to the experimenter.

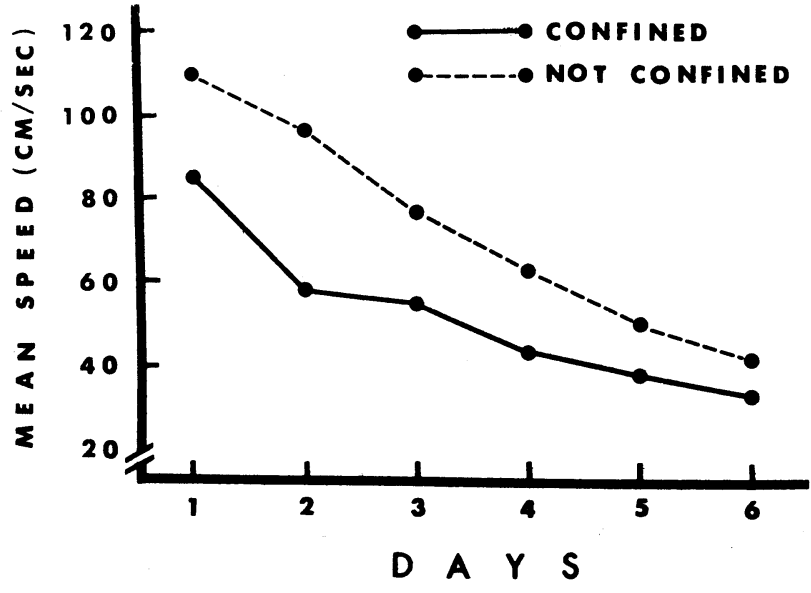

Figure 3. Total alley extinction speeds as a function of confinement.

The present results do not support the contention of McCain and Bowen (1967) that constant delay results in increased persistence of the running response. In this regard, our results are consistent with the large majority of studies reviewed by Campbell and Knouse (1972). Apparently, constant delay can, under some unknown circumstances, result in increased persistence. We had thought that perhaps the brevity of acquisition training used by McCain and Bowen had been responsible. Our data suggest otherwise unless our 10 training trials are beyond the limit of brief-training established by McCain and Bowen's use of five trials. A close inspection of our data suggests that 10 trials may have been too many to replicate the McCain and Bowen extinction effect. Our constant delay subjects began to slow down after about six training trials. Had we begun extinction after only five trials, as did McCain and Bowen, we too might have found that constant delay led to faster responses during extinction.

\section{REFERENCES}

Campbell, P. E.. \& Knouse, S. B. Extinction following delayed reward: A review. Psychonomic Monograph Supplements. 1973, 4. 257-268.

Crum, J.. Brown, W. L.. \& Bitterman, M. E. The effects of partial and delayed reinforcement on resistance to extinction. American Journal of Psychology. 1951. 64. 288-297.

Howlett, J. C.. \& Sheldon, M. H. Effects of partial delay of reinforcement following a small number of acquisition trials. Psychonomic Science. 1968, 11, 259.

McCain. G.. \& Bowen, J. Pre- and post-reinforcement delay with a small number of acquisition trials. Psychonomic Science, 1967. 7. 121-122.

RENNER, K. E. Delay of reinforcement: A historical review. Psychological Bulletin, 1964, 61, 341-361.

RobBins, D. Partial reinforcement: A selective review of the alleyway literature since 1960. Psychological Bulletin. 1971, 76. 415-431.

Wike, E. L.. Mellgren, R. I., \& Wike, S. S. Runway performance as a function of delayed reinforcement and delay. box continement. Psychological Record. 1968, 18, 9-18.

(Received for publication May 21. 1975.) 